\title{
Two crystal structures demonstrate large conformational changes in the eukaryotic ribosomal translocase
}

Rene Jørgensen, Pedro A Ortiz, Anne Carr-Schmid, Poul Nissen, Terri Goss Kinzy \& Gregers Rom Andersen Nat. Struct. Biol. 10, 379-385 (2003).

Some of the amino acid residues in Figs. 2 and 3 of this paper were incorrectly labeled. In Fig. 2a, residue 535 should be a glutamate (E535); in Fig. 2d, residue 191 should be a threonine (T191) and residue 192 should be a tyrosine (Y192); and in Fig. 3a, residue 798 should be a phenylalanine (Phe798). The panels with corrected labels are printed here. The authors apologize for any inconvenience this may have caused.

$2 a$

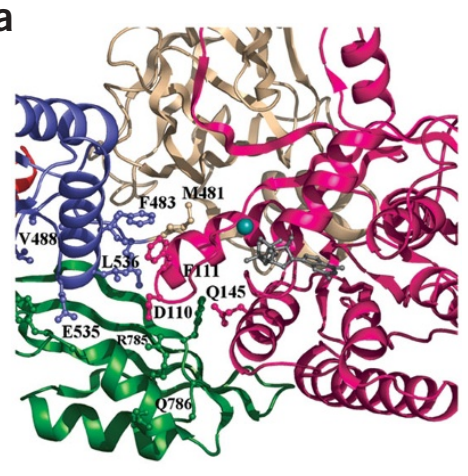

2d

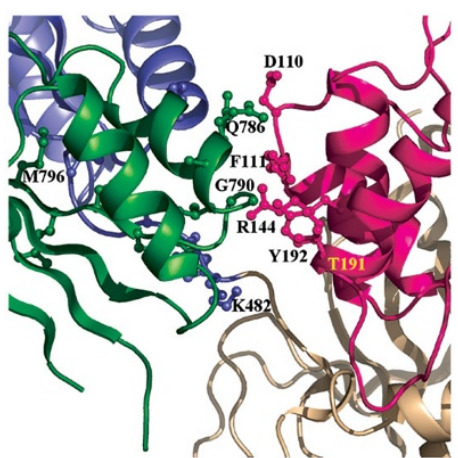

$3 a$

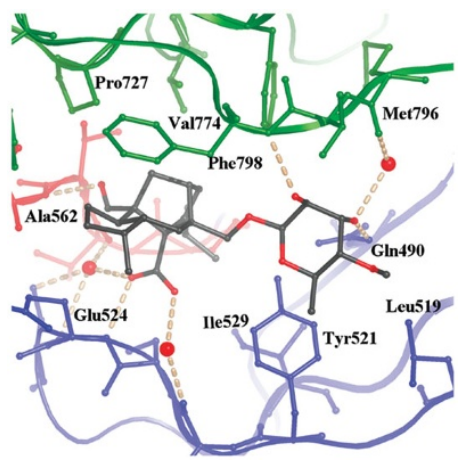

\section{Structural basis of heroin and cocaine metabolism by a promiscuous} human drug-processing enzyme

Sompop Bencharit, Christopher L Morton, Yu Xue, Philip M Potter \& Matthew R Redinbo Nat. Struct. Biol. 10, 349-356 (2003).

In the original print version of this manuscript, Fig. 1 contained several errors. In Fig. 1b, a double bond is missing in the structures of heroin, 6-acetylmorphine and morphine. In Fig. 1c, the $N$-alkyl group is one carbon too short in the naloxone methiodide structure. In addition, the stereochemistry of the structures in Fig. $1 \mathrm{~b}$ and of naloxone methiodide in Fig. 1c was incorrect. The errors occurred during preparation of these panels for publication, and the authors apologize for any inconvenience this may have caused. Corrected versions of both panels are reproduced here.

$1 b$

Hydrolysis of heroin

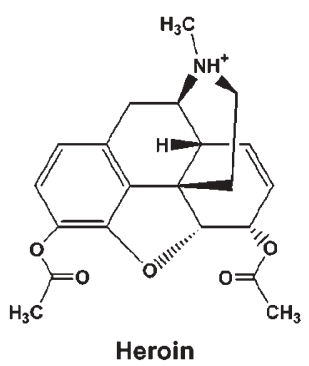

$1 c$<smiles>COC(=O)C1CC2CCC(C1)C2C(=O)Oc1ccccc1</smiles>

S-Cocaine
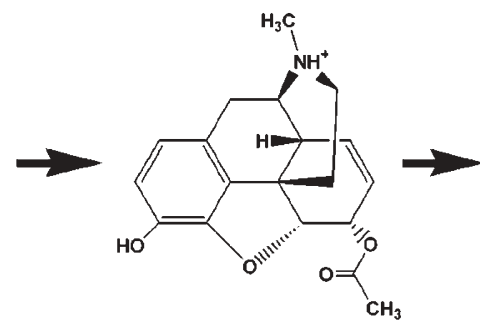

6-Acetyl morphine

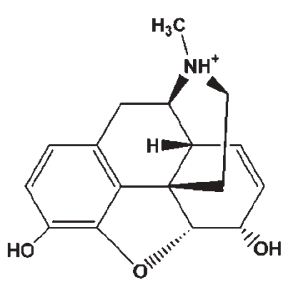

Morphine

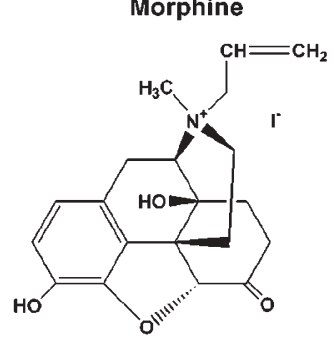

Naloxone methiodide 